\title{
Rare Case of Isolated Plasmodium Vivax Malaria Presenting with Pancytopenia: A Case Report
}

\author{
Khalid Shahzad ${ }^{1}$, Anum Ashfaq ${ }^{1 *}$, Muhammad Atif Beg², Tariq Hussain ${ }^{1}$, Uzma Batool ${ }^{2}$, Syed Yar \\ Muhammad Shah ${ }^{3}$, Zala Ejaz ${ }^{4}$, Mahzaib Raza ${ }^{4}$, Ramshaw Waqar ${ }^{4}$ and Zunaira Habib \\ ${ }^{1}$ Resident General Medicine, Pakistan Atomic Energy Commission General Hospital, Pakistan \\ ${ }^{2}$ Consultant General Medicine, Pakistan Atomic Energy Commission General Hospital, Pakistan \\ ${ }^{3}$ Resident General Medicine, Ayub Teaching Hospital, Pakistan \\ ${ }^{4}$ Resident General Medicine, Pakistan Atomic Energy Commission General Hospital, Pakistan \\ *Corresponding author: Anum Ashfaq, Resident General Medicine, Pakistan Atomic Energy Commission General Hospital, \\ Islamabad, Pakistan
}

\section{ARTICLE INFO}

Received: 幽 August 12, 2021

Published: 幽 September 17, 2021

Citation: Khalid Shahzad, Anum Ashfaq, Muhammad Atif Beg, Tariq Hussain, Uzma Batool, et al., Rare Case of Isolated Plasmodium Vivax Malaria Presenting with Pancytopenia: A Case Report. Biomed J Sci \& Tech Res 38(5)-2021. BJSTR. MS.ID.006208.

Abbreviations: LDH: Lactate Dehydrogenase; TLC: Total Leucocyte Count; G6PD: Glucose-6-Phospate Dehydrogenase; ARDS: Acute Respiratory Distress Syndrome; HLH: Hemophagocytic Lymphohistiocytosis; TNF: Tumor Necrosis Factor; IL: Interleukin

\begin{abstract}
Pancytopenia is a triad of simultaneous drop in all the three major blood cells including Hemoglobin, White blood cells and Platelets. Etiology of pancytopenia is diverse comprising both hematopoietic and non-hematopoietic disorders including various infections. Malaria is a rare cause of pancytopenia which mostly occur with Plasmodium falciparum and very few cases reported with Plasmodium vivax. This is a case of a 17-year-old girl with no previous co-morbids presented with high grade fever, vomiting, loose motions and decrease appetite for 5 days. On examination she was dehydrated and pale with bruising on arms and thighs. Spleen was palpable. Rest of systemic examination was unremarkable. Complete blood picture showed decreased TLC, Low Hemoglobin and Thrombocytopenia. Liver function tests, Renal function tests, Serum electrolytes, Urine routine examination and Erythrocyte sedimentation rate were normal. C-reactive protein, LDH, Ferritin and D-dimers were raised. Peripheral film showed Retic count of 3\%. Dengue serology and Covid-19 PCR was negative. Blood and Urine Cultures showed no growth. Ultrasound abdomen showed splenomegaly. Peripheral smear for Malarial Parasite showed early trophozoites of Plasmodium vivax. Patient was started on Intravenous Artesunate as she could not tolerate Oral antimalarial. Patient became afebrile and blood counts improved after few days and was discharged with follow up test for G6PD assay.
\end{abstract}

Keywords: Pancytopenia; Plasmodium vivax; Splenomegaly; Artesunate

\section{Background}

Plasmodium vivax is one of the most widely distributed specie of genus plasmodium causing infection in humans with approximately 80 million new cases annually. Although severe infection with $P$. vivax is extremely rare Kochar et al has reported a case of $P$. vivax presenting with severe infection leading to severe anemia, renal involvement and ARDS with multiorgan failure. Pancytopenia is an extremely rare complication of $P$. vivax malaria with various proposed mechanisms including macroangiopathic hemolytic anemia, hemophagocytic syndrome and direct bone marrow suppression [1]. This is one such case of isolated vivax malaria presenting with pancytopenia

\section{Case Presentation}

A 17-year-old girl with no previous co-morbids presented with high grade fever associated with rigors and chills, vomiting, loose motions and decrease appetite for 5 days. On examination 
patient had Bp 100/70, Pulse 92/min, Temperature 101F, Oxygen saturation $98 \%$ with room air. She was dehydrated and pale with bruises on arms and thighs. There was no jaundice or lymph nodes. On abdominal examination Abdomen was soft non-tender with palpable spleen with a span of about 2 fingers breath below the costal margin. Liver was not palpable. Cardiovascular and Respiratory examination was unremarkable. Complete blood picture showed TLC 2740/microliter, Hemoglobin $10.4 \mathrm{~g} / \mathrm{dl}$ and Platelets 16000/microliter. Liver function tests, Renal function tests, Serum electrolytes, Urine routine examination and Erythrocyte sedimentation rate were normal. C-reactive protein was $48, \mathrm{LDH}$ 443, Ferritin 1021 and D-dimers were 890. Peripheral film showed Retic count of 3\%. Dengue serology and Covid-19 PCR was negative. Blood and Urine Cultures showed no growth. Ultrasound abdomen showed splenomegaly with size of $13 \mathrm{~cm}$. Peripheral smear for Malarial Parasite showed early trophozoites of Plasmodium vivax. Patient was started on Intravenous Artesunate as she could not tolerate Oral anti-malarial. Her Hematologic parameters dropped further during her stay at the hospital with TLC to 2520/ microliter, $\mathrm{Hb}$ to $8.5 \mathrm{~g} / \mathrm{dl}$ and platelets to $11000 /$ microliter. Patient responded well to Intravenous artesunate. She became afebrile and her blood parameters normalized after 5 days. She was discharged with follow up test for G6PD assay after one week.

\section{Discussion}

Malaria is a worldwide national health problem in many countries, and many occur in tropical and subtropical areas including sub-Saharan Africa, Asia and Latin America [2]. Worldwide approximately 3 billion people resides in areas which have high risk of malaria transmission. 1.1 to 2.7 million deaths occur due to severe malaria every year. Malaria is considered as a 5 th leading cause of death due to infectious disease and $2^{\text {nd }}$ leading causes of death in Africa with 1 million people dying every year in Africa. About $85 \%$ of all the malarial cases in the world are due to Plasmodium falciparum with Plasmodium vivax on $2^{\text {nd }}$ number. $90 \%$ of all the malarial deaths occur in Africa [3].

Ofall the plasmodium species, the deadliestand the most virulent is $P$. falciparum which can lead to life threating complications and death if not treated early. Species other than $P$. falciparum usually cause mild disease with minimal complications [2]. P. vivax malaria is a parasitic infection which is carried by Anopheles mosquito [4]. Life cycle of $P$. vivax is a unique phase known as hypnozoite stage in which the parasite remains dormant in the liver causing frequent relapses after acute infection is treated. Severe complications due to $P$. vivax are extremely rare compared to P. falciparum [5].

Hematological changes occurring in malaria include anemia, thrombocytopenia, leucopenia, neutropenia, leukocytosis, atypical leukocytosis and splenomegaly. One study has shown that lymphopenia, leucopenia and thrombocytopenia are the key predictors of malaria infection. Low $\mathrm{Hb}$, High lymphocyte count, low platelets and monocytosis are more severe in chronic malaria compared to acute malaria [6]. Hematologic changes due to malaria depend on various factors including malaria endemicity, background hemoglobin disorders, demographic factors and level of malarial immunity. Thrombocytopenia and anemia are the main blood abnormalities occurring in malaria [3]. Pancytopenia is an uncommon manifestation of malaria and is mostly common with P. falciparum and occur due to both direct and indirect effect of infection on hematopoietic cells in the bone marrow [7]. Two main mechanisms involved in pancytopenia involve direct bone marrow suppression and hemophagocytosis, the latter is mostly reported with P. falciparum malaria.

P. vivax has lesser pyrogenic threshold, marked inflammatory response and very high cytokine production as compared to $P$. falciparum. There have been case reports of severe disseminated infection with multi organ failure due to P. vivax. Studies have also shown that $P$. vivax can lead to acute tubular necrosis and acute interstitial nephritis termed as Malarial Nephropathy [1].

Pancytopenia secondary to P. vivax malaria is extremely rare and has only been reported in $0.9 \%$ of the confirmed $P$. vivax cases. To the best of author's knowledge, only up to 5 cases of isolated $P$. vivax malaria with pancytopenia have been reported in literature without other associated comorbids [2,5]. Key mechanism involved in the development of pancytopenia in P. vivax is microangiopathic hemolytic anemia followed by hemophagocytic syndrome which has rarely been reported with P. vivax [5]. Hemophagocytic syndrome is an unusual clinicopathological syndrome that is characterized by overt immunological responses by T-cells producing high levels of interferon gamma, TNF alpha, IL-1, IL-2 and IL-18 leading to activation of macrophages which in turn causes phagocytosis of hematopoietic cells and bone marrow suppression. It can be fatal if misdiagnosed or diagnosis is delayed.

Hemophagocytic syndrome mainly occurs secondary to many infections including viral, bacterial, fungal and parasitic infections [4]. Estimation of exact prevalence of malaria associated HLH is very difficult because bone marrow biopsy is not routinely performed to diagnose malarial infection and exact mortality rate is also not low. It can be fatal if not treated. Malaria associated phagocytic syndrome can lead to prolong hemophagocytosis which is a very rare complication and can lead to prolong anemia. It has been reported only with $P$. falciparum and not with $P$. vivax [2]. Various treatment modalities to treat HLH include treating the causative agent, immunosuppressant, steroids and IV immunoglobulins [8]. Various studies have proven that malaria associated HLH has an excellent response to antimalarials and supportive care without any need for immunosuppressants and steroids $[2,8]$. 
Malaria must always be kept in differential diagnosis as a cause of prolong fever with refractory anemia and pancytopenia particularly in endemic areas and asymptomatic patient despite of negative smear and rapid antigen test. Bone marrow biopsy is the key to diagnose $P$. falciparum malaria in such cases $[9,10]$.

\section{Conclusion}

Malaria is one of many infections which can involve any organ of the body especially the bone marrow. Bone marrow involvement causes decrease in all the three cell lines leading to pancytopenia. It is important that malaria should always be included in the differential diagnosis whenever pancytopenia is worked up because it is one of the treatable causes of pancytopenia with excellent prognosis.

\section{Conflict of Interest}

No conflict of interest with any institution/organization.

Grant or Fellowship Supporting the Writing of Paper

Nil.

\section{Institutional Approval}

Institutional approval was obtained in compliance with regulation of our institution and generally accepted guidelines governing such work.

\section{Processing Fee}

Nil.

\section{Author's Contribution}

Author fulfilling the ICMJE criteria.

ISSN: 2574-1241

DOI: $10.26717 /$ BJSTR.2021.38.006208

Anum Ashfaq. Biomed J Sci \& Tech Res

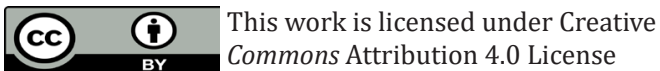

Submission Link: https://biomedres.us/submit-manuscript.php

\section{References}

1. Mitra A, Ray S (2020) Acute Kidney Injury and Pancytopenia, A Rare Case Presentation of Vivax Malaria. J Med Case Rep Case Series.

2. Sharma S, Dawson L (2020) Pancytopenia induced by secondary hemophagocytic lymphohistiocytosis: A rare, overlooked dreadful complication of Plasmodium vivax. Trop Parasitol.

3. Awoke N, Arota A (2019) Profiles of hematological parameters in Plasmodium falciparum and Plasmodium vivax malaria patients attending Tercha General Hospital, Dawuro Zone, South Ethiopia. Infect Drug Resist.

4. Albaker W (2009) Acute Plasmodium vivax malaria presenting with pancytopenia secondary to hemophagocytic syndrome: case report and literature review. J Family Community Med.

5. Kumar R, Sharma A (2017) Acute pancytopenia secondary to hemophagocytic syndrome due to Plasmodium vivax malaria with chloroquine treatment failure: Case report. Int J Adv Med.

6. Omarine Nlinwe N, Nange TB (2020) Assessment of Hematological Parameters in Malaria, among Adult Patients Attending the Bamenda Regional Hospital. Anemia.

7. Anabire NG, Aryee PA, Helegbe GK (2018) Hematological abnormalities in patients with malaria and typhoid in Tamale Metropolis of Ghana. BMC Res Notes.

8. Chaudhry S, Arya A, Matlani M, Singh V, Meena SS (2021) Pancytopenia with hemophagocytic lymphohistiocytosis in Plasmodium falciparum: A unusual presentation. Trop Parasitol.

9. Aggarwal V, Maheshwari A, Rath B, Kumar P, Basu S (2011) Refractory pancytopenia and megaloblastic anemia due to falciparum malaria. J Trop Pediatr.

10. Hayat Z, Ullah I, Hayat K, Khan HA, Raziq F, et al. (2019) Refractory Anemia and Pancytopenia as Presentations of Falciparum Malaria in Population of Khyber Pakhtunkhwa, Pakistan. Gomal Journal of Medical Sciences.

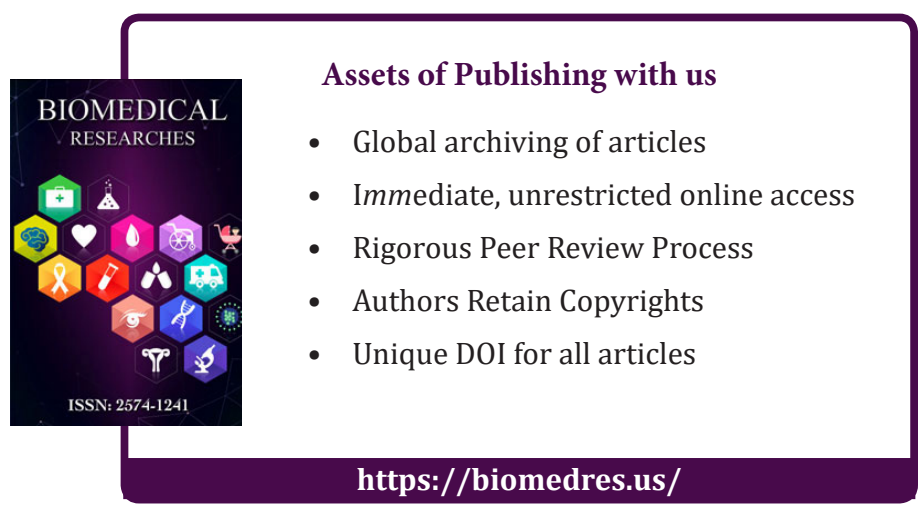

